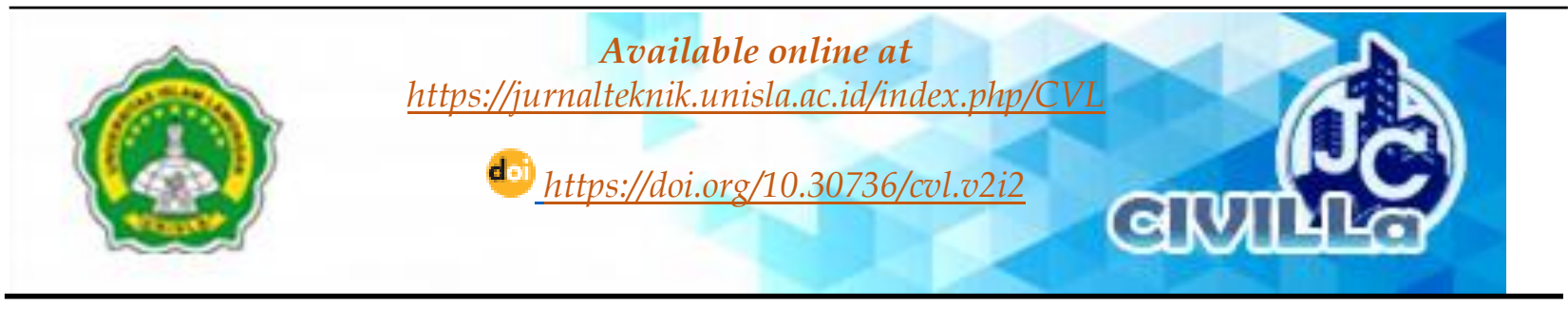

\title{
Gable Frame Structure Planning Using LRFD Method In Pamekasan Factor Warehouse Project
}

\author{
Ahmad Ridwan ${ }^{1}$, Nur Indah Mukhoyyaroh ${ }^{2}$ \\ ${ }^{1}$ Civil Engineering, Universitas Kadiri. \\ ${ }^{2}$ Civil Engineering, Universitas Islam Lamongan

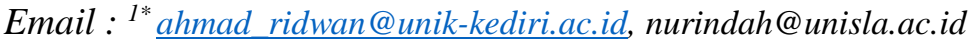

\section{A R T I C LE IN F O}

\section{Article History :}

Article entry : 2021-10-03

Article revised : 2021-12-14

Article received : 2021-12-16

\section{Keywords :}

Roof Construction, LRFD,

Gable Frame, Steel Frame

\section{IEEE Style in citing this} article :

A. Ridwan and N. I. Mukhoyyaroh, "Gable Frame Structure Planning Using Lrfd Method in Pamekasan Factory Warehouse Project," CIVILA, vol. 6, no. 2, pp. 225-244, 2021.

\begin{abstract}
A B S T R A C T
Currently, the use of steel as a building construction has been widely used as the main material for building structures. Steel frames come in a variety of profiles and sizes. The use of steel frames can be adjusted to the type of construction to be built. From the results of the planning of the WF steel roof structure on the factory warehouse construction project in Pamekasan, it was obtained planning data: Gording using Profile C $125 \times 50 \times 40 \times 4,5$. Trekstang uses $8 \mathrm{~mm}$ diameter, Wind ties use $10 \mathrm{~mm}$ diameter steel, Rafter uses WF 350x350x19x19 profile, column uses WF 350x350x19x19 profile, 8 pieces A325 bolts with $22 \mathrm{~mm}$ diameter, Hoist Crane Beam uses IWF Bulit-Up beam with $600 \times 1144 \times 18 \times 22$ profile, Base Plate uses a size of 500x500x8mm with a column of $600 \times 600$. Calculation using LRFD is very important to get a structure that is stable, strong enough, serviceable, durable, and economical. A structure is said to be stable if it is not easily overturned, tilted, or displaced during the design of the building.
\end{abstract} print, search, or link to the full texts of its articles and allow readers to use them for any other lawful purpose. 


\section{Introduction}

Currently, the use of steel as a building construction framework has been widely used as the main material for building structures[1][2]. Considering the use of wood, which is considered expensive compared to steel, it becomes the best alternative to building frames[3][4].

The advantage of using steel frames for building construction is that it is easy to install, practical, strong and durable. Besides steel will not be affected by extreme weather changes. Steel can be designed to be not easy to rust, mushroom[5][6]. In addition, it can be made of steel that is waterproof, termite-resistant, not easily porous and has great yield strength[7][8]. Steel frames are not only used for roof construction, but can also be used to build large buildings, such as factories, warehouses, construction structures, etc.

Calculation using LRFD is very important to get a structure that is stable, strong enough, serviceable, durable, and economical. A structure is said to be stable if it is not easily overturned, tilted, or displaced during the design of the building.

Other mechanical properties of structural steel for planning purposes are determined as follows (SNI 1729-2015) [9] :

- Modulus of elasticity : $\mathrm{E}=2100000 \mathrm{MPa}$

- Shear modulus $\quad: \mathrm{G}=80.000 \mathrm{MPa}$

- Poisson's ratio $\quad: \mu=0,3$

- Expansion coefficient : á $=12$ x $10-6 /{ }^{0} \mathrm{C}$

\section{Research Method}

\subsection{Description and Technical}

\section{Planning Engineering Data}

1. The span length of the factory warehouse (distance between columns) is $30 \mathrm{~m}$.

2. The length of the building is $80 \mathrm{~m}$, the height is $5 \mathrm{~m}$, the angle of inclination is $20^{\circ}$, the distance between the horses is $4 \mathrm{~m}$.

3. Crane load $P 10$ tons, wind load $40 \mathrm{~kg} / \mathrm{m} 2$, roof type Galvalume $(12 \mathrm{~kg} / \mathrm{m} 2$

4. Wind bonding (bracing) of the wall of the stiffening frame, the side walls are exposed.

5. Steel quality A36 ( $\mathrm{fy}=240 \mathrm{Mpa})$, bolt connection type A325.

6. Design using an easel.

Table 1. Load Combination Process

\begin{tabular}{|l|c|c|c|c|c|c|}
\hline \multirow{2}{*}{ Load Combination } & \multicolumn{2}{|c|}{ Equally } & \multicolumn{2}{c|}{$\begin{array}{c}\text { Load } \\
\text { Combination }\end{array}$} & \multicolumn{2}{c|}{ Centered } \\
\cline { 2 - 3 } & $\mathrm{x}$ & $\mathrm{y}$ & \multicolumn{2}{c|}{$\mathrm{x}$} & $\mathrm{y}$ \\
\hline $1,4 \mathrm{D}$ & 14,112 & 38,78 & & & & \\
\hline $1,2 \mathrm{D}+1,6 \mathrm{~L}+0,5$ (Lr or S or R) & 63,936 & 183,59 & $1,6 \mathrm{~L}$ & & 51,84 & 150,35 \\
\hline
\end{tabular}


Civilla: Jurnal Teknik Sipil Universitas Islam Lamongan

Volume 06 Number 2 Year 2021

Volume 06 Number 2 Year 2021
\begin{tabular}{|l|l|l|l|l|c|c|}
$1,2 \mathrm{D}+1,6(\mathrm{Lr}$ or S or R) + (L or \\
$0.5 \mathrm{~W})$
\end{tabular}

Source : Analysis Results (2021)

\section{Trekstang Planning}

\section{Loading}

Dead Load (D)

Curtain weight $=\quad 8.32 \mathrm{~kg} / \mathrm{m} \times 2 \times 10$ $=166.4 \mathrm{~kg}$

Roof covering $=\quad 12 \mathrm{~kg} / \mathrm{m}^{2} \times 1.54 \mathrm{m \times} 4$ $=73.92 \mathrm{~kg}$

Connection weight $=\quad 10 \% \times 73.92 \mathrm{~kg}$$$
=7.39 \mathrm{~kg}
$$

D total $=247.71 \mathrm{~kg}$

\section{Loading Combination}

$\mathrm{D} \quad=247.71 \mathrm{~kg}$

$\mathrm{L} \quad=100 \mathrm{~kg}$

Choose the biggest combination

$\mathrm{Pu}=1,2 \mathrm{D}+1,6 \mathrm{~L}+0,5(\mathrm{Lr}$ or $\mathrm{S}$ or $\mathrm{R}) \quad=457,252 \mathrm{~kg}$

$\mathrm{Pu}=457,252 \mathrm{~kg}$.

\section{Trekstang}

Trekstang is used to reduce the deflection of the $\mathrm{x}$-axis direction (roof slope)[10], so that the force acting is the load in the $\mathrm{x}$-axis direction. Working style:

$\mathrm{P}_{\mathrm{ux}} \quad=\mathrm{P}_{\mathrm{u}} \sin 30^{\circ}=790,28 \mathrm{~kg}$

The biggest force is on the top of the handlebar (near the cam) of $790.28 \mathrm{~kg}$. This style will be used in the planning of the Trestang dimension[11].

\section{Trekstang Design}

quality of steel used A36/BJ-37

Minimum yield stress, fy $=240 \mathrm{Mpa}$

Minimum breaking stress, $\mathrm{fu}=370 \mathrm{Mpa}$

Trekstang is used to withstand tensile loads, so the design of the handlebars uses tensile analysis[12][13].

At yielding, the nominal resistance of the tension bar is:

$T_{n} \quad=\varnothing A_{g} f_{y}$

$7902,8=0,9 A_{g}(240)$ 
$A_{g} \quad=36,58 \mathrm{~mm}^{2}$

Then the required diameter of the tension rod is:

$A_{g} \quad=0,25 \pi \mathrm{d}^{2}$

$\mathrm{d} \quad=6,82 \mathrm{~mm}$

$>$ In the fracture condition, the nominal resistance of the tension member is:

$T_{n}=\varnothing A_{g} f_{u}$

$7902,8=0,75 A_{g}(370)$

$A_{g} \quad=28,47 \mathrm{~mm}^{2}$

Then the required diameter of the tension rod is:

$A_{g} \quad=0,25 \pi \mathrm{d}^{2}$

$\mathrm{d}=6,02 \mathrm{~mm}$

So the diameter of the handlebar used is $8 \mathrm{~mm}$.

\section{Wind Ties Calculation}

The load is planned as a concentrated load at each wind bond joint as follows:

\section{Dead Load $\left(\mathbf{P}_{\mathbf{D}}\right)$}

D $\quad(12 \times 4 \times 1.54)+(8,32+10 \% \times 8.32) \times 4)$

Live Load $\left(\mathbf{P}_{\mathrm{L}}\right)$

$$
=110.528 \mathrm{~kg}
$$$$
=100 \mathrm{~kg}
$$

\section{Load in the direction of the axis of the rod}

$\mathrm{P}_{\mathrm{D}}=\frac{110.528}{\sin (56.65)} \times 2=265.69 \mathrm{~kg}$

$\mathrm{P}_{\mathrm{L}}=\frac{100}{\sin (56.65)} \times 2=239.52 \mathrm{~kg}$

\section{Ultimate Total Load}

$$
\mathrm{P}_{\mathrm{u}} \quad=1.2 \mathrm{P}_{\mathrm{D}}+1.6 \mathrm{P}_{\mathrm{L}}
$$

$$
=7020.6 \mathrm{~N}
$$

\section{Wind Tensile Prisoner}

The wind bond is assumed to be $10 \mathrm{~mm}$ so that the tensile resistance of the bar at yielding is:

$$
\begin{aligned}
\mathrm{T}_{\mathrm{n}} & =\mathrm{A}_{\mathrm{g}} \mathrm{f}_{\mathrm{y}} \\
& =1 / 4 \times \pi \times 10^{2} \times 240=18840 \mathrm{~N}
\end{aligned}
$$

The tensile resistance of the bar at fracture is:

$$
\begin{aligned}
\mathrm{T}_{\mathrm{n}} & =\mathrm{A}_{\mathrm{g}} \mathrm{f}_{\mathrm{u}} \\
& =1 / 4 \times \pi \times 10^{2} \times 370=29045 \mathrm{~N}
\end{aligned}
$$

Tensile resistance at yielding condition is less than tensile resistance under fracture condition, so Tn at yielding condition is more decisive [14][15].

\section{Diameter of Wind Ties Used}

$\mathrm{T}_{\mathrm{u}}<\varnothing \mathrm{T}_{\mathrm{n}}$

$7020,6 \mathrm{~N}<16956 \mathrm{~N}$ 


\section{Gable Frame Planning (Non Sway Assumption)}

Dead Load (D)

- Roof covering weight 1,54 x 4 x 12

- Block's own weight $156 \times 15,43$

- Curtain's own weight 8,32 x 80

- Wind bond weight $0,0005 \times 7850$

- Connection weight $10 \%$ x $(2189,985)$
$=73,92 \mathrm{~kg}$

$=1450,42 \mathrm{~kg}$

$=665,6 \mathrm{~kg}$

$=0,045 \mathrm{~kg}$

$=218,99 \mathrm{~kg}$

$D$ total $=2408,975 \mathrm{~kg}$

\section{Live Load $(\mathbf{L})$}

Table 3. Loading Combination

\begin{tabular}{|l|l|}
\hline $1,4 \mathrm{D}$ & 3372,57 \\
\hline $1,2 \mathrm{D}+1,6 \mathrm{~L}+0,5(\mathrm{Lr}$ or $\mathrm{S}$ or $\mathrm{R})$ & 3050,77 \\
\hline $1,2 \mathrm{D}+1,6(\mathrm{Lr}$ or $\mathrm{S}$ or $\mathrm{R})+(\mathrm{L}$ or $0.5 \mathrm{~W})$ & 2992,37 \\
\hline $1,2 \mathrm{D}+1,0 \mathrm{~W}+\mathrm{L}+0,5(\mathrm{Lr}$ or $\mathrm{S}$ or $\mathrm{R})$ & 2990,77 \\
\hline $1,2 \mathrm{D}+1,0 \mathrm{E}+\mathrm{L}+0,2 \mathrm{~S}$ & 2990,77 \\
\hline $0,9 \mathrm{D}+1,0 \mathrm{~W}$ & 2168,08 \\
\hline $0,9 \mathrm{D}+1,0 \mathrm{E}$ & 2168,08 \\
\hline
\end{tabular}

A. Non Sway Field Image

Momen

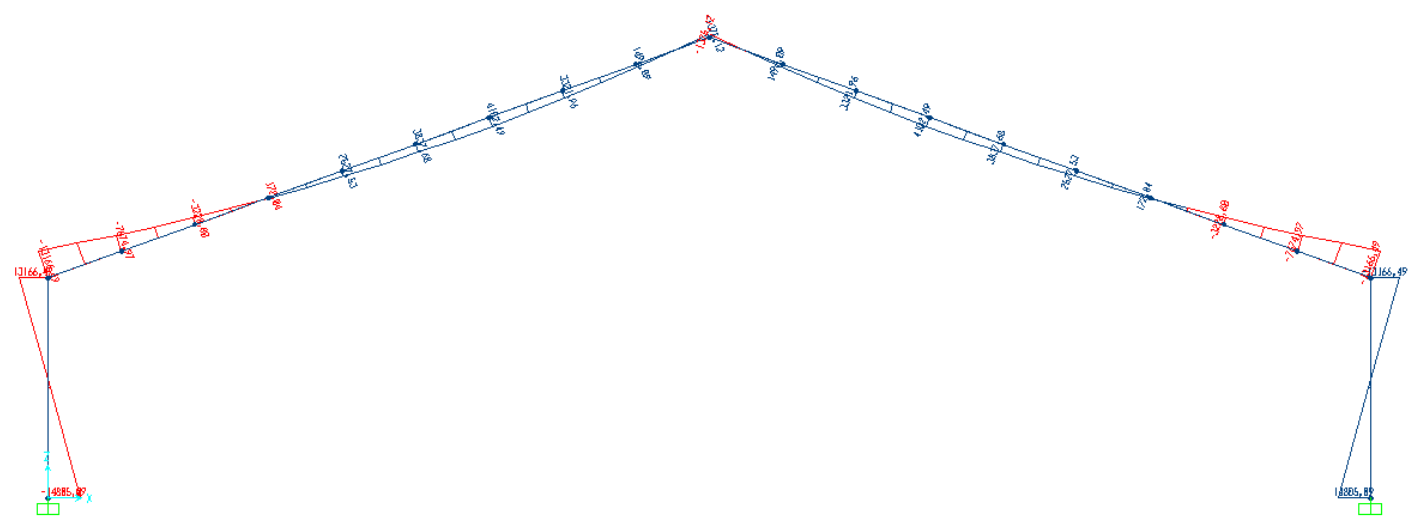


B. Slide

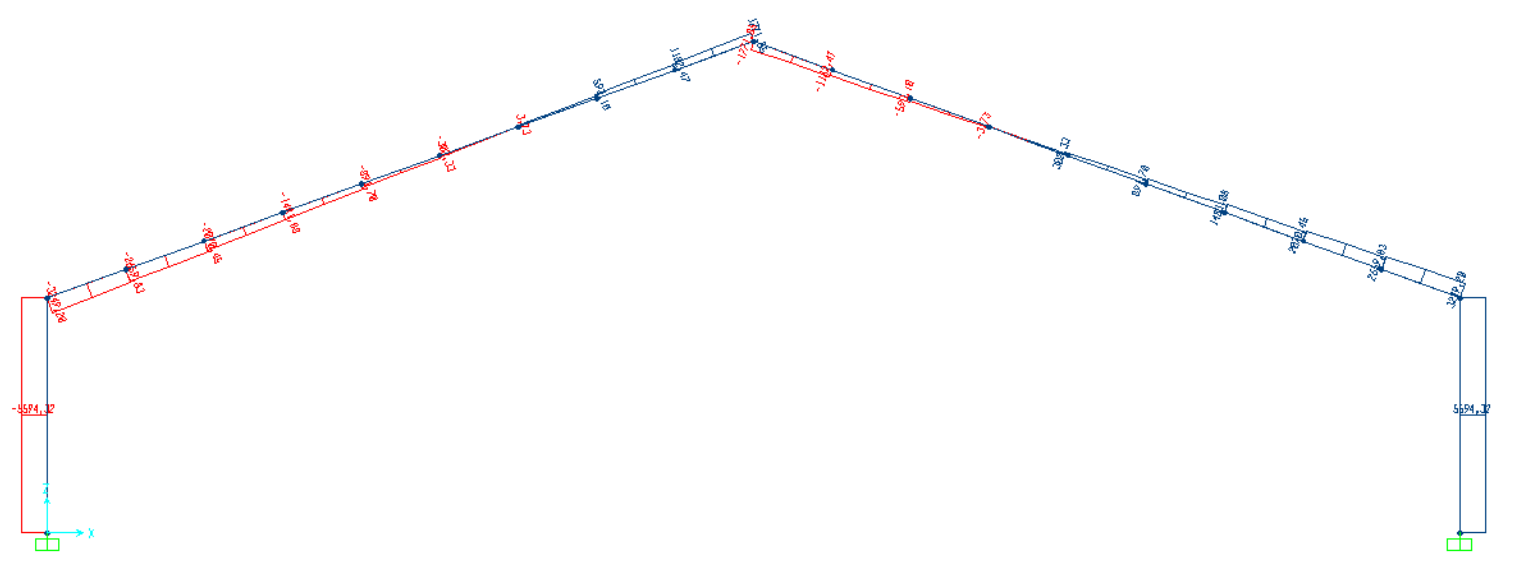

C. Axial

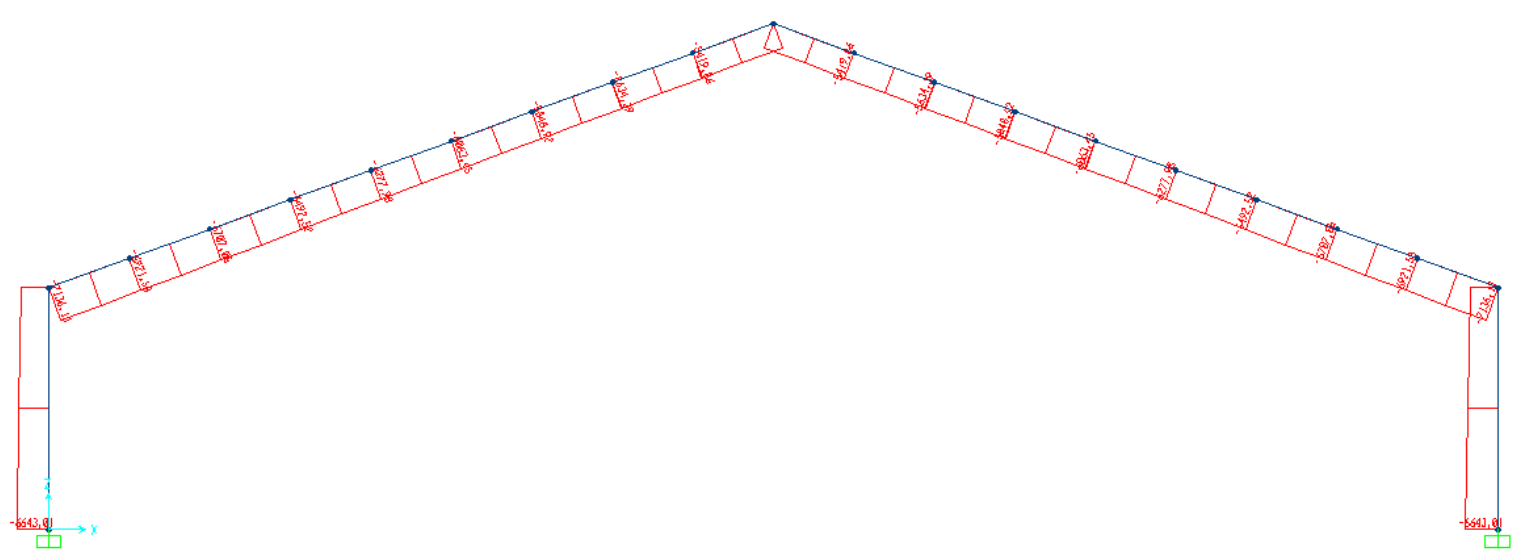

\section{Calculation of the Non-Sway Assumption Rafter}

$\begin{array}{llll}\mathrm{M} 1 & =13166,49 \mathrm{kgm} & \mathrm{M} 2 & =172,04 \mathrm{kgm} \\ \mathrm{V} 1 & =7136,11 \mathrm{~kg} & \mathrm{~V} 2 & =5419,86 \mathrm{~kg}\end{array}$

For the calculation of the rafter, the largest forces are used:

$\mathrm{Pu} \quad=7136,11 \mathrm{~kg}$

$\mathrm{M} \quad=13166,49 \mathrm{kgm}$

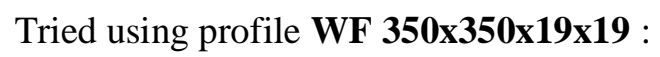

Stiffness: 
$\mathrm{GA}=\frac{\sum \frac{I}{\text { LRafter }}}{\sum_{\text {LColumn }}}=0,324$

$\mathrm{GB}=\frac{\sum \frac{I}{\frac{L}{\text { LRafter }}}}{\sum \frac{I}{\text { LRafter }}}=1$

Table 4. monogram effective length factor $\mathrm{k}$ for frame

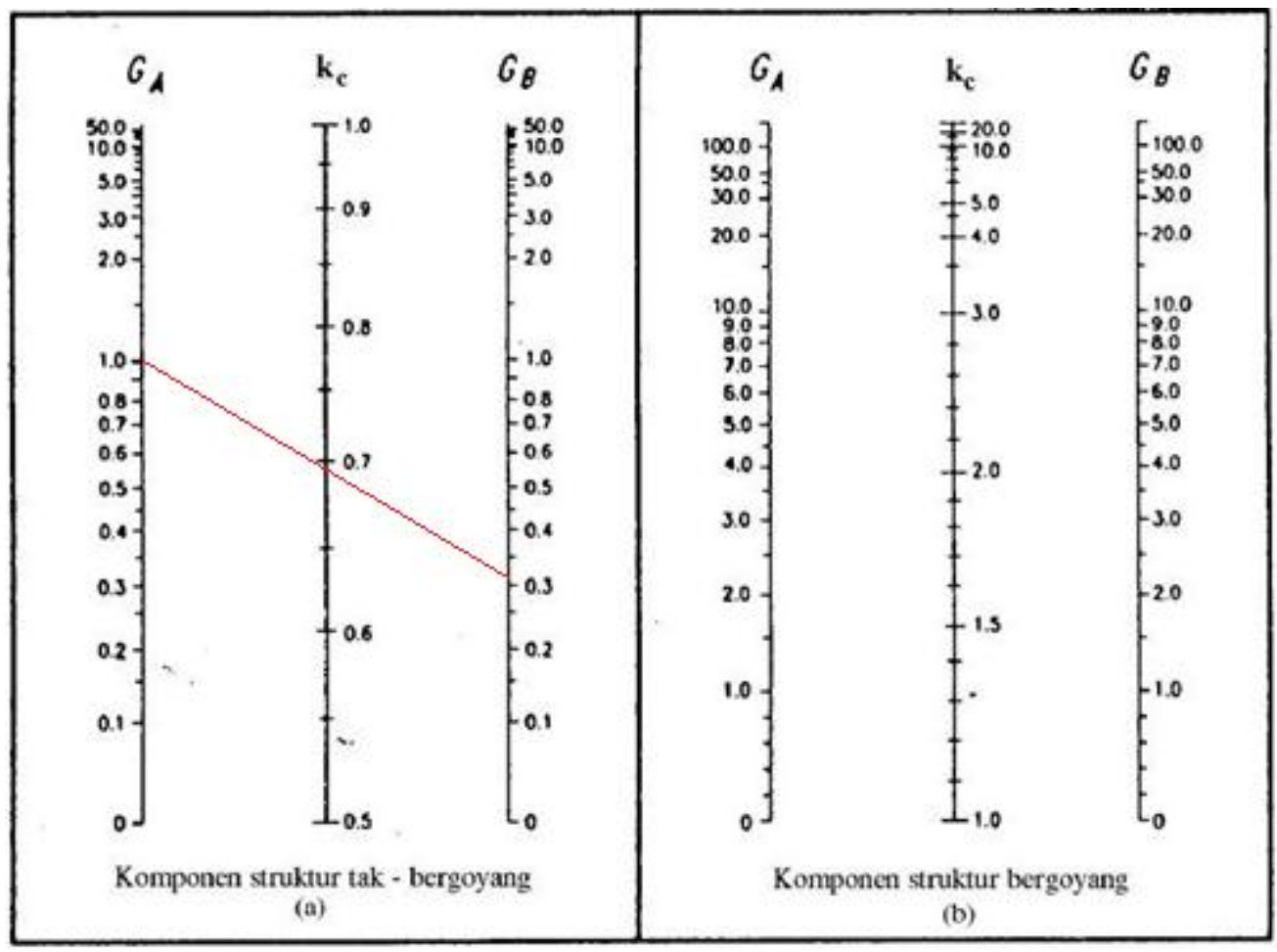

Based on the picture, get the value of KK $\quad=0,67$

$\mathrm{KL} \quad=10,34=1033,81 \mathrm{~cm}=10338,1 \mathrm{~mm}$

$\mathrm{KL} / \mathrm{r}_{\mathrm{y}}=121,19 \mathrm{~cm}=1211,9 \mathrm{~mm}$

$\mathrm{KL} / \mathrm{r}_{\mathrm{x}}=70,32 \mathrm{~cm}=703,2 \mathrm{~mm}$

Tried using profile WF 350x350x19x19 with $\mathrm{Ag}=198,4 \mathrm{~cm}^{2}=19840 \mathrm{~mm}^{2}$

Score $P_{n}$, according to SNI 1729-2015, chapter E. 3 is:

$\mathrm{P}_{\mathrm{n}}=\mathrm{F}_{\mathrm{cr}} \cdot \mathrm{A}_{\mathrm{g}}$

a. If $\frac{K L}{r} \leq 4,71 \sqrt{\frac{E}{F y}}$ or $\frac{F_{y}}{F_{e}} \leq 2,25$

$\mathrm{F}_{\mathrm{cr}}=\left[0,658^{\frac{F_{y}}{F_{e}}}\right] \mathrm{F}_{\mathrm{y}} \quad 1 . \mathrm{a}$

b. If $\frac{K L}{r}>4,71 \sqrt{\frac{E}{F y}}$ or $\frac{F_{y}}{F_{e}}>2,25$

$\mathrm{F}_{\mathrm{cr}}=0,877 F_{e} \quad 1 . \mathrm{b}$ 
$\mathrm{Fe}=\frac{\pi^{2} \mathrm{E}}{\left(\frac{K L}{r}\right)^{2}}$

Equation 1.b is used because

$\frac{K L}{r}>4,71 \sqrt{\frac{E}{F y}}$

$703,22>135,96$

$\mathrm{F}_{\mathrm{e}}=\frac{\pi^{2} 200000}{\left(\frac{10338,1}{147}\right)^{2}}=\frac{1971920}{4944,90}=398,77$

So $\mathrm{F}_{\mathrm{cr}}=0,877 \times 398,77$

$$
=349,72 \mathrm{MPa}
$$

So as, $\mathrm{P}_{\mathrm{n}}=\mathrm{F}_{\mathrm{cr}} \mathrm{X} \mathrm{A}_{\mathrm{g}}=349,72.19840=6938444,8 \mathrm{~N}$

\section{Check cross-sectional compactness}

$$
\begin{array}{ll}
\lambda & =\mathrm{bf} / 2 \mathrm{tf}=357 / 2 \times 19 \quad=9,39 \\
\lambda_{\mathrm{p}} & =0,38 \sqrt{\frac{E}{F y}}=10,97 \\
\lambda_{\mathrm{r}} & =1,0 \sqrt{\frac{E}{F y}}=26,86
\end{array}
$$

Check,

$\lambda \leq \lambda_{\mathrm{p}}$

$9,39 \leq 10,97 \quad \rightarrow$ compact

$\mathrm{Mn}=\mathrm{Mp}=\mathrm{f}_{\mathrm{y}} \cdot \mathrm{z}_{\mathrm{x}}=240 \cdot 2450000=588000000 \mathrm{Nmm}$

$\mathrm{Pu} / \varnothing \mathrm{Pn} \quad<\quad 0,2$

$71361,1 / 5897678,08<0,2$

$0,012<0,2 \ldots \ldots . .0 K$

\section{Check Lateral Bend}

$$
\begin{aligned}
& \mathrm{P}_{\mathrm{u}}=7136,11 \mathrm{~kg}=71361,1 \mathrm{~N} \\
& \mathrm{P}_{\mathrm{n}}=6938444,8 \mathrm{~N}=693844,48 \mathrm{~kg} \\
& \mathrm{M}_{\mathrm{ux}}=13166,49 \mathrm{kgm} \\
& \mathrm{M}_{\mathrm{p}}=588000000 \mathrm{Nmm}=58800 \mathrm{kgm} \\
& \mathrm{M}_{\mathrm{nx}}=\mathrm{Z}_{\mathrm{x}} \cdot \mathrm{f}_{\mathrm{y}} \\
&=5880000 \mathrm{kgcm} \\
& \mathrm{M}_{\mathrm{ny}}=\mathrm{Z}_{\mathrm{y}} \cdot \mathrm{f}_{\mathrm{y}} \\
&=1941600 \mathrm{kgcm} \\
& \frac{P_{u}}{2 . \varnothing . P_{n}}+\left\{\frac{M_{u x}}{\varnothing . M_{n x}}+\frac{M_{u y}}{\varnothing . M_{n y}}\right\} \leq 1 \\
& 0,254 \leq 1 \ldots . . .0 K ! ! !
\end{aligned}
$$




\section{Calculation of Non Sway Assumption Column}

$\begin{array}{llll}\mathrm{M} 1 & =14805,09 \mathrm{kgm} & \mathrm{M} 2 & =13166,49 \mathrm{kgm} \\ \mathrm{V} 1 & =6643,01 \mathrm{~kg} & \mathrm{~V} 2 & =5724,84 \mathrm{~kg}\end{array}$

For the calculation of the column the largest forces are used:

$\mathrm{Pu} \quad=6643,01 \mathrm{~kg}$

$\mathrm{M} \quad=14805,09 \mathrm{kgm}$

Tried using profile WF 350x350x19x19 :

Stiffness:

$\begin{aligned} \text { GA } & =\frac{\sum \frac{I}{\text { LRafter }}}{\sum_{\text {LColumn }}}=3,086 \\ \mathrm{~GB} & =\frac{\sum \frac{I}{\text { LRafter }}}{\sum_{\text {LRafter }}}=1\end{aligned}$

Table 5. monogram effective length factor $\mathrm{k}$ for frame

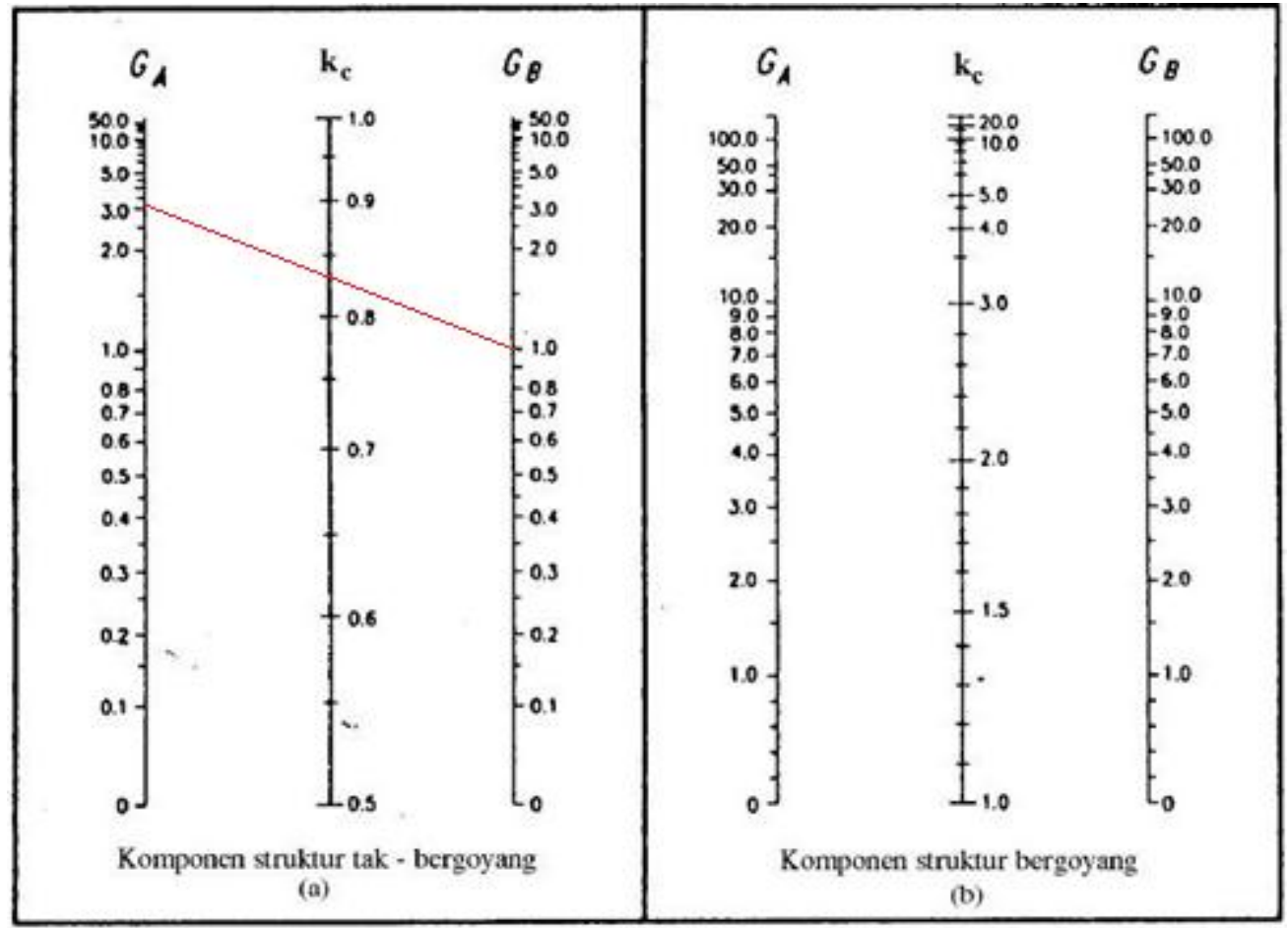

Based on the picture, get the value of $\mathrm{K}$

$\mathrm{K} \quad=0,83$

$\mathrm{KL} \quad=12,80=1280 \mathrm{~cm}=12800 \mathrm{~mm}$

$\mathrm{KL} / \mathrm{r}_{\mathrm{y}}=150,06 \mathrm{~cm}=1500,6 \mathrm{~mm}$

$\mathrm{KL} / \mathrm{r}_{\mathrm{x}}=87,07 \mathrm{~cm}=870,7 \mathrm{~mm}$

Tried using profile WF 350x350x19x19 with $\mathrm{Ag}=198,4 \mathrm{~cm}^{2}=19840 \mathrm{~mm}^{2}$

Equation 1.b is used because 
$\frac{K L}{r}>4,71 \sqrt{\frac{E}{F y}}$

$870,7>135,96$

$\mathrm{F}_{\mathrm{e}}=\frac{\pi^{2} 200000}{\left(\frac{12800}{147}\right)^{2}}=\frac{1971920}{7581,18}=260,89$

So $\mathrm{F}_{\mathrm{cr}}=0,877 \times 260,89$

$$
=228,80
$$

So as, $\mathrm{P}_{\mathrm{n}}=\mathrm{F}_{\mathrm{cr}}$ x A $A_{\mathrm{g}}=228,80.19840=4539392 \mathrm{~N}$

\section{Check cross-sectional compactness}

$\lambda \quad=\mathrm{bf} / 2 \mathrm{tf}=357 / 2 \times 19=9,39$

$\lambda_{\mathrm{p}} \quad=0,38 \sqrt{\frac{E}{F y}} \quad=10,97$

$\lambda_{\mathrm{r}} \quad=1,0 \sqrt{\frac{E}{F y}} \quad=26,86$

Check,

\begin{tabular}{|c|c|c|c|c|}
\hline$\lambda$ & $\leq$ & \multicolumn{3}{|c|}{$\lambda_{\mathrm{p}}$} \\
\hline 9,39 & $\leq$ & 10,97 & & $\rightarrow$ compact \\
\hline $\mathrm{Mn}$ & $=$ & \multicolumn{3}{|c|}{$\mathrm{Mp}=\mathrm{f}_{\mathrm{y}} \cdot \mathrm{z}_{\mathrm{x}}=240.2450000=588000000 \mathrm{Nmm}$} \\
\hline $\mathrm{Pu} / \varnothing$ & ?n & $<$ & 0,2 & \\
\hline \multicolumn{3}{|c|}{$66430,1 / 385848,32$} & $<$ & 0,2 \\
\hline \multicolumn{3}{|l|}{0,17} & $<$ & $0,2 \ldots . . . . \mathrm{OK}$ \\
\hline
\end{tabular}

\section{Check Lateral Bend}

$\mathrm{P}_{\mathrm{u}} \quad=6643,01 \mathrm{~kg}$

$\mathrm{P}_{\mathrm{n}} \quad=4539392 \mathrm{~N}=453939,2 \mathrm{~kg}$

$\mathrm{Mu}_{\mathrm{x}} \quad=14805,09 \mathrm{kgm}$

$\mathrm{M}_{\mathrm{p}} \quad=588000000 \mathrm{Nmm}=58800 \mathrm{kgm}$

$\mathrm{M}_{\mathrm{nx}} \quad=\mathrm{Z}_{\mathrm{x}} \cdot \mathrm{f}_{\mathrm{y}}$

$=5880000 \mathrm{kgcm} \quad=\quad 58800 \mathrm{kgm}$

$\mathrm{M}_{\mathrm{ny}} \quad=\mathrm{Z}_{\mathrm{y}} \cdot \mathrm{f}_{\mathrm{y}}$

$=1941600 \mathrm{kgcm} \quad=\quad 19416 \mathrm{kgm}$

$\frac{P_{u}}{2 . \emptyset \cdot P_{n}}+\left\{\frac{M_{u x}}{\varnothing \cdot M_{n x}}+\frac{M_{u y}}{\emptyset \cdot M_{n y}}\right\} \leq 1$

$0,287 \leq 1$...... OK $! ! !$

\section{Connection Planning}

\section{Rafter Bolt and Column Planning For Profile 350x350x19x19}

From the calculation results SAP2000v14 obtained :

$\mathrm{M}_{\mathrm{u}} \quad=13166,49 \mathrm{kgm}=1316649 \mathrm{kgcm}$ 
$\mathrm{Pu} \quad=7136,11 \mathrm{~kg}$

Bolts are used A325 with :

Threaded bolt $\varnothing 7 / 8^{\prime \prime}=22 \mathrm{~mm}=2,2 \mathrm{~cm}$

$\mathrm{A}_{\mathrm{b}} \quad=\pi \mathrm{r}^{2}=\pi\left(\frac{2,2}{2}\right)^{2}=3,454 \mathrm{~cm}^{2}$

Plate thickness $(\mathrm{tp})=12 \mathrm{~mm}$

High quality bolt

$$
\begin{array}{rl}
\mathrm{f}_{\mathrm{ub}} 825 \mathrm{Mpa} & =8250 \mathrm{~kg} / \mathrm{cm}^{2} \\
\mathrm{f}_{\mathrm{yb}} & 585 \mathrm{Mpa}=5850 \mathrm{~kg} / \mathrm{cm}^{2}
\end{array}
$$

\section{Rafter Bolt Plan}

Calculation of shear strength of one bolt

$$
\begin{aligned}
\varnothing R n v & =0,75 \times \mathrm{r}_{1} \times \mathrm{f}_{\mathrm{ub}} \times \mathrm{A}_{\mathrm{b}} \\
& =10685,81 \mathrm{~kg}
\end{aligned}
$$

Calculation of the strength to support one for

$$
\begin{aligned}
\varnothing \mathrm{Rn} & =0,75 \times 2,4 \times \mathrm{d} \times \mathrm{tp} \times \mathrm{f}_{\mathrm{ub}} \\
& =39204 \mathrm{~kg}
\end{aligned}
$$

Calculation of tensile strength of one bolt

$$
\begin{aligned}
\varnothing \mathrm{Rnt} & =0,75 \times 0,75 \times \mathrm{fu} \times \mathrm{Ab} \\
& =16028,72 \mathrm{~kg}
\end{aligned}
$$

Assumed number of bolts

It is assumed that there are 8 bolts

Edge distance (S1)

$$
=1,25 \times \mathrm{db}=1,25 \times 22=27,5 \mathrm{~mm}
$$

Distance between bolts (S)

$$
=3 \quad x \mathrm{db}=3 \quad \mathrm{x} 22=66 \mathrm{~mm}
$$

$\mathrm{S} 1=100 \mathrm{~mm}$

$\mathrm{S} \quad=100 \mathrm{~mm}$

Ruv $=P u / n=7136,11 / 8=829,01 \mathrm{~kg}$

$\left(\frac{R_{u v}}{\emptyset R_{n v}}\right)^{2}+\left(\frac{R_{u t}}{R_{n t}}\right)^{2} \leq 1$

$\frac{829,01}{10685,81}+\frac{R_{u t}}{16028,72} \leq 1$

$\mathrm{R}_{\mathrm{ut}}=\mathrm{T}=14785,2 \mathrm{~kg}$

Connection moment

$\mathrm{a}=\left(\frac{\sum T}{f y \times B}\right)=\frac{14785,2 \times 6}{5850 \times 35}=0,43 \mathrm{~cm}$

$\mathrm{d} 1=(100 / 10)-0,43=9,57 \mathrm{~cm}$

$\mathrm{d} 2=\mathrm{d} 1+100 / 10=19,57 \mathrm{~cm}$

$\mathrm{d} 3=\mathrm{d} 2+100 / 10=29,57 \mathrm{~cm}$

$\Sigma \mathrm{d}=58,71 \mathrm{~cm}$

$\varnothing \mathrm{Mn}=\frac{0,9 \times f y x a^{2} x B}{2}+\Sigma \mathrm{dT}$ 


$$
\begin{aligned}
& =\frac{0,9 \times 5850 \times 0,43^{2} \times 35}{2}+58,71 \times 14785,2 \times 2 \\
& =17036,22+1736078,184 \\
& =1753114,404 \mathrm{kgcm}>1316649 \mathrm{kgcm} \text {...... OK !!! }
\end{aligned}
$$

From the calculation results SAP2000v14 obtained :

$\mathrm{M}_{\mathrm{u}} \quad=14805,09 \mathrm{kgm}=1480509 \mathrm{kgcm}$

$\mathrm{Pu} \quad=6643,01 \mathrm{~kg}$

Used A325 bolts with:

Threaded bolt $\varnothing 7 / 8^{\prime \prime}=22 \mathrm{~mm}=2,2 \mathrm{~cm}$

$\mathrm{A}_{\mathrm{b}} \quad=\pi \mathrm{r}^{2}=\pi\left(\frac{2,2}{2}\right)^{2}=3,454 \mathrm{~cm}^{2}$

Plate thickness $(\mathrm{tp})=12 \mathrm{~mm}$

High quality bolt $\quad \mathrm{f}_{\mathrm{ub}} 825 \mathrm{Mpa}=8250 \mathrm{~kg} / \mathrm{cm}^{2}$

$$
\mathrm{f}_{\mathrm{yb}} 585 \mathrm{Mpa}=5850 \mathrm{~kg} / \mathrm{cm}^{2}
$$

\section{Rafter Column Bolt Plan}

Calculation of shear strength of one bolt

$$
\begin{aligned}
\varnothing \mathrm{Rnv} & =0,75 \times \mathrm{r}_{1} \times \mathrm{f}_{\mathrm{ub}} \times \mathrm{A}_{\mathrm{b}} \\
& =10685,81 \mathrm{~kg}
\end{aligned}
$$

Calculation of the bearing strength of one bolt

$$
\begin{aligned}
\varnothing \mathrm{Rn} & =0,75 \times 2,4 \times \mathrm{d} \times \mathrm{tp} \times \mathrm{f}_{\mathrm{ub}} \\
& =39204 \mathrm{~kg}
\end{aligned}
$$

Calculation of tensile strength of one bolt

$$
\begin{aligned}
\varnothing \mathrm{Rnt} & =0,75 \times 0,75 \times \mathrm{fu} \times \mathrm{Ab} \\
& =16028,72 \mathrm{~kg}
\end{aligned}
$$

It is assumed that there are 8 bolts

Edge distance (S1) $=1,25 \times \mathrm{db}=1,25 \times 22=27,5 \mathrm{~mm}$

Distance between bolts $(\mathrm{S}) \quad=3 \quad \mathrm{x} \mathrm{db}=3 \quad \mathrm{x} 22=66 \mathrm{~mm}$

$\mathrm{S} 1=100 \mathrm{~mm}$

$\mathrm{S} \quad=100 \mathrm{~mm}$

$\mathrm{R}_{\mathrm{uv}}=\mathrm{Pu} / \mathrm{n}=6643,01 / 8=830,38 \mathrm{~kg}$

$\left(\frac{R_{u v}}{\emptyset R_{n v}}\right)^{2}+\left(\frac{R_{u t}}{R_{n t}}\right)^{2} \leq 1$

$\frac{830,38}{10685,81}+\frac{R_{u t}}{16028,72} \leq 1$

$\mathrm{R}_{\mathrm{ut}}=\mathrm{T}=14783,15 \mathrm{~kg}$

Connection moment

$\mathrm{a}=\left(\frac{\sum T}{\text { fy } \times B}\right)=\frac{14783,15 \times 6}{5850 \times 35}=0,43 \mathrm{~cm}$

$\mathrm{d} 1=(100 / 10)-0,43=9,57 \mathrm{~cm}$

$\mathrm{d} 2=\mathrm{d} 1+100 / 10=19,57 \mathrm{~cm}$ 
$\mathrm{d} 3=\mathrm{d} 2+100 / 10=29,57 \mathrm{~cm}$

$$
\Sigma \mathrm{d}=58,71 \mathrm{~cm}
$$

$$
\begin{aligned}
\varnothing \mathrm{Mn} & =\frac{0,9 \times f y \times a^{2} \times B}{2}+\Sigma \mathrm{dT} \\
& =\frac{0,9 \times 5850 \times 0,43^{2} \times 35}{2}+58,71 \times 14783,15 \times 2 \\
& =17036,22+1735837,473 \\
& =1752873,693 \mathrm{kgcm}>1316649 \mathrm{kgcm} \ldots . . . \text { OK } ! ! !
\end{aligned}
$$

\section{Crane Structure Planning}

\section{Structural Geometry}

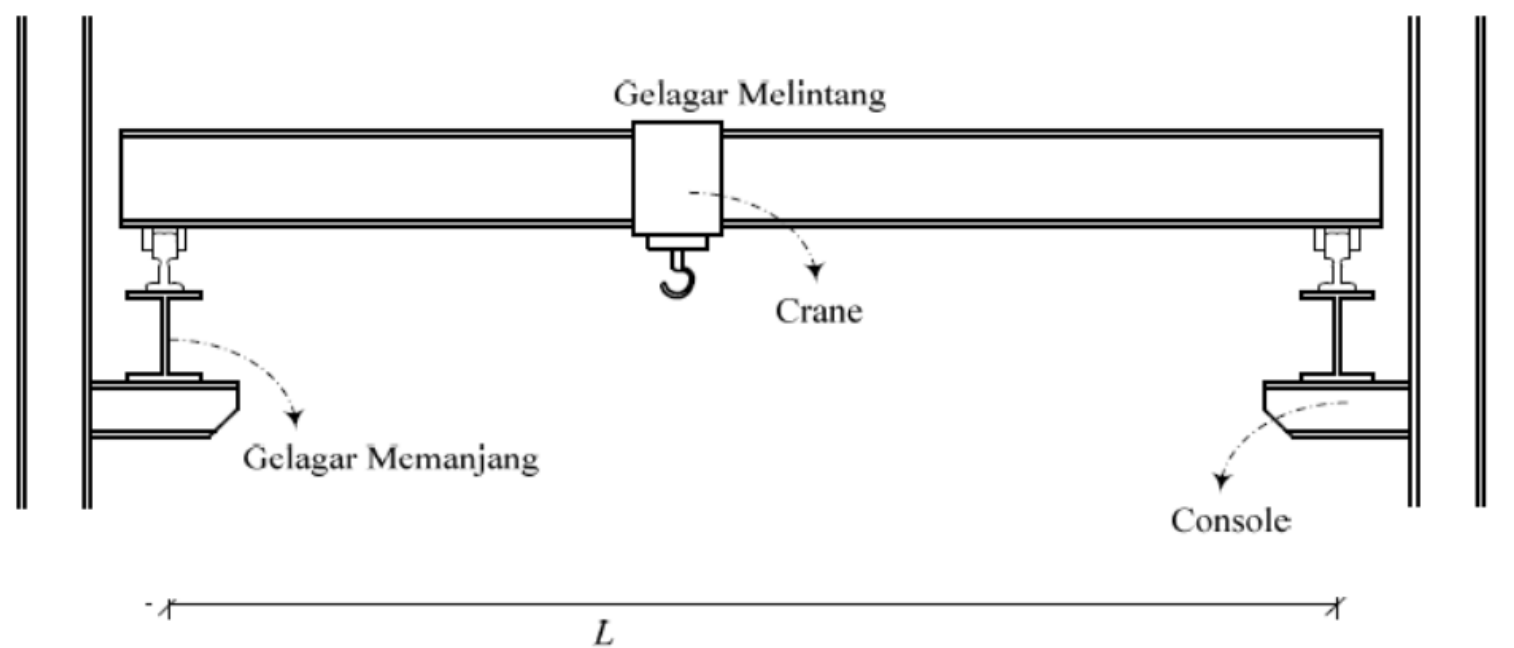

Figure 1. Bridge Beam Design

The length of the bridge beam that will be reviewed is 30 meters and 80 meters.

\section{Material Data}

The steel material used in the design of this steel structure is Hot-Rolled steel (WF, C, T profiles and steel plates) with the following data:

- Melting strength $\quad: \mathrm{F}_{\mathrm{y}} \quad=240 \mathrm{MPa}$

- Melting strength $\quad \mathrm{E} \quad=200000 \mathrm{MPa}$

- Shear Modulus $\quad: \mathrm{G} \quad=80000 \mathrm{MPa}$

\section{Loading}

The loads that are reviewed as design loads in the calculation of this factory warehouse structure are: Dead Load which includes the self-weight of the steel profile with a steel density ( $\gamma$ steel) of 7850 $\mathrm{kg} / \mathrm{m} 3$ as listed in Table 3-1 SNI 03-1727- 1989-F and Live Load which includes crane loads (Pcrane) of 10 tons $=10000 \mathrm{~kg}$. [16][17][18] 


\section{Hoist Crane Beam Planning of 30 meters}

\section{Hoist Crane Beam Planning}

In this plan, 2 girders are used bridge beam

Built-up IWF profile data:

$\mathrm{B}=600 \mathrm{~mm}$

$\mathrm{H}=1144 \mathrm{~mm}$

$\mathrm{H}_{\mathrm{w}}=1100 \mathrm{~mm}$

$\mathrm{t}_{\mathrm{w}}=18 \mathrm{~mm}$

$\mathrm{t}_{\mathrm{f}}=22 \mathrm{~mm}$

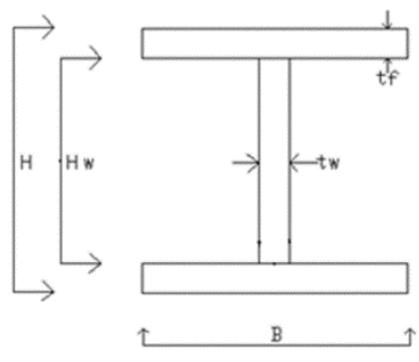

Figure 2. Hoist Crane

- The cross-sectional property data are as follows:
$\mathrm{A} \quad=\left(2 \cdot \mathrm{t}_{\mathrm{f}} \cdot \mathrm{B}\right)+\left(\mathrm{t}_{\mathrm{w}} \cdot \mathrm{H}_{\mathrm{w}}\right)$
$=46200 \mathrm{~mm}^{2}$
$\mathrm{q}=\mathrm{A} \cdot \gamma_{\text {baja }}$
$=362,67 \mathrm{~kg} / \mathrm{m}$
$\mathrm{I}_{\mathrm{x}} \quad=2\left\{\left[\frac{1}{12} \cdot B \cdot t_{f}{ }^{3}\right]+\left[t_{f} \cdot B \cdot\left(\frac{H-t_{f}}{2}\right)^{2}\right]\right\}+\left\{\frac{1}{12} \cdot t_{w} \cdot H_{w}{ }^{3}\right\}=6,15 \cdot 10^{9} \mathrm{~mm}^{4}$
$\mathrm{I}_{\mathrm{y}} \quad=\frac{1}{12}\left[\left(2 \cdot t_{f} \cdot B^{3}\right)+\left(H \cdot t_{W}{ }^{3}\right)\right]$
$=7,93 \cdot 10^{8} \mathrm{~mm}^{4}$
$\mathrm{S}_{\mathrm{x}} \quad=\frac{I_{X}}{0,5 \cdot H}$
$=1,07 \cdot 10^{7} \mathrm{~mm}^{3}$
$\mathrm{S}_{\mathrm{Y}} \quad=\frac{I_{Y}}{0,5 \cdot H}$
$=2,64 \cdot 10^{6} \mathrm{~mm}^{3}$
$\mathrm{Z}_{\mathrm{x}}=\left[B \cdot t_{f} \cdot\left(H-t_{f}\right)\right]+\left(\frac{1}{4} \cdot t_{w} \cdot H_{w}{ }^{2}\right)$
$=2,02 \cdot 10^{7} \mathrm{~mm}^{3}$
$\mathrm{Z}_{\mathrm{y}} \quad=1,5 \cdot \mathrm{S}_{\mathrm{y}}$
$=3,96 \cdot 10^{6} \mathrm{~mm}^{3}$
$\mathrm{J} \quad=\left(t_{w}{ }^{3} \cdot H_{w}\right)+\left(2 \cdot t_{f}{ }^{3} \cdot B\right)$
$=1,19 \cdot 10^{7} \mathrm{~mm}^{4}$

- Calculation of the forces in the ultimate on the hoist-crane beam:

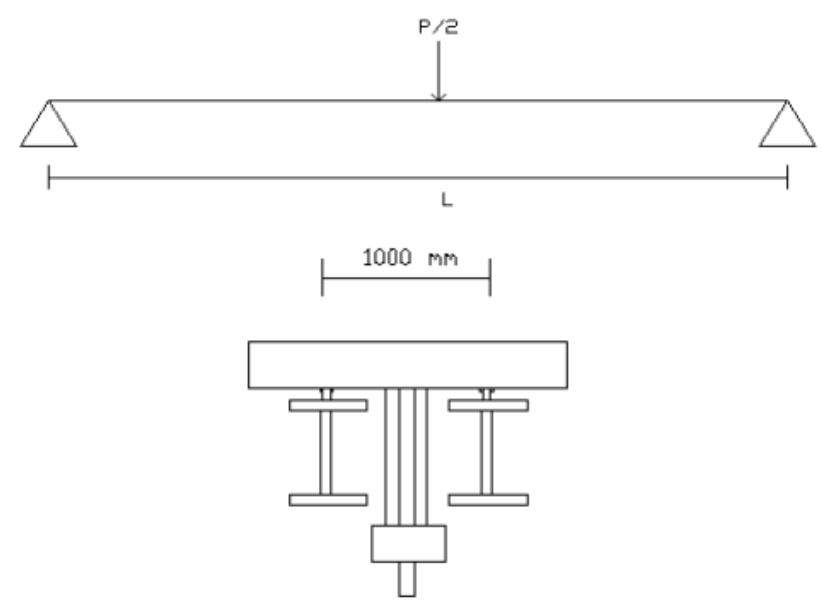

Figure 3. Hoist Crane
Hoist-crane span length : $\mathrm{L}=30 \mathrm{~m}$
$=30000 \mathrm{~mm}$
Crane load on 1 beam $: P=5$ Ton
$=5000 \mathrm{~kg}$
Shock factor (impact factor)

$$
=1,25
$$


Civilla: Jurnal Teknik Sipil Universitas Islam Lamongan

Volume 06 Number 2 Year 2021

Crane load design

$$
: \mathrm{P}=5 \text { Ton } .1,25=6,25 \text { Ton }
$$

Ultimate moment:

$$
\begin{aligned}
\mathrm{M}_{\mathrm{DL}}=1,2 \cdot \frac{1}{8} \cdot \mathrm{q} \cdot \mathrm{L}^{2} \\
=48960,45 \mathrm{kgm} \\
\mathrm{M}_{\mathrm{LL}} \quad=1,6 \cdot \frac{1}{4} \cdot \mathrm{P} \cdot \mathrm{L} \\
=75000 \mathrm{kgm} \\
\mathrm{M}_{\mathrm{u}} \quad=\mathrm{M}_{\mathrm{DL}}+\mathrm{M}_{\mathrm{LL}} \\
=123960,45 \mathrm{kgm}=1239,6045 \mathrm{kNm}
\end{aligned}
$$

Maximum shear force due to crane load:

$$
\begin{aligned}
\mathrm{V}_{\mathrm{DL}} & =1,2\left(\mathrm{q}_{\mathrm{u}} \cdot \mathrm{L}\right) \\
& =13056,12 \mathrm{~kg} \\
\mathrm{~V}_{\mathrm{LL}} & =1,6 \cdot \frac{P}{2} \\
& =10000 \mathrm{~kg} \\
\mathrm{~V}_{\mathrm{u}} & =\mathrm{V}_{\mathrm{DL}}+\mathrm{V}_{\mathrm{LL}} \\
& =23056,12 \mathrm{~kg}
\end{aligned}
$$

- Check the Slimness of the Cross

1. Wing cross section

Check cross section of the wing

$$
\begin{array}{ll}
\lambda & =\mathrm{bf} / 2 \mathrm{tf}=27,2727 \\
\lambda_{\mathrm{p}} & =0,38 \sqrt{\frac{E}{F y}}=10,97 \\
\lambda_{\mathrm{r}} & =1,0 \sqrt{\frac{E}{F y}}=26,86
\end{array}
$$

Check,

$$
\begin{array}{llll}
\lambda & \leq & \lambda_{\mathrm{p}} & \\
27,2727 & \leq & 10,97 & \rightarrow \text { Not compact }
\end{array}
$$

2. Body Cross

$$
\begin{array}{ll}
\lambda & =\mathrm{bf} / 2 \mathrm{tf}=61,11 \\
\lambda_{\mathrm{p}} & =3,76 \sqrt{\frac{E}{F y}}=108,54 \\
\lambda_{\mathrm{r}} & =5,70 \sqrt{\frac{E}{F y}}=164,54
\end{array}
$$

Check,

$\lambda \quad \leq \quad \lambda_{\mathrm{p}}$




\section{$61,11 \leq 108,54 \rightarrow$ Compact}

- Lateral Bending Parameter

Finding Value $\mathrm{L}_{\mathrm{p}}$

$$
\begin{aligned}
& \mathrm{r}_{\mathrm{y}} \quad=\sqrt{\frac{I_{y}}{A}}=\sqrt{\frac{793000000}{46200}}=130,975 \mathrm{~mm} \\
& \mathrm{~L}_{\mathrm{p}} \quad=1,76 \cdot \mathrm{r}_{\mathrm{y}} \cdot \sqrt{\frac{E}{F y}} \\
&=1,76 \cdot 130,975 \cdot \sqrt{\frac{200000}{240}} \\
&=6654,419 \mathrm{~mm}
\end{aligned}
$$

Finding Value $\mathrm{L}_{\mathrm{r}}$

$$
\begin{aligned}
& \text { Ho }=\mathrm{H}-\mathrm{t}_{\mathrm{f}}=1144-22=1122 \mathrm{~mm} \\
& \begin{aligned}
\mathrm{C}_{\mathrm{w}} & =\frac{1}{4} \cdot I_{y} \cdot H_{o}{ }^{2} \\
& =2,49 \cdot 10^{14} \mathrm{~mm}^{6} \\
& =\sqrt{\frac{\sqrt{I y \cdot C w}}{S_{x}}}=\sqrt{\frac{\sqrt{7,93 \cdot 10^{8} \times 2,49 \cdot 10^{14}}}{1,07 \cdot 10^{7}}}=203,322
\end{aligned}
\end{aligned}
$$

$\mathrm{C}$ for double symmetrical I profile $\quad=1$

$\mathrm{L}_{\mathrm{r}} \quad=1,95 \mathrm{r}_{\mathrm{ts}} \frac{E}{0,7 \cdot f_{y}} \sqrt{\frac{J x C}{S_{x} h_{o}}+\sqrt{\frac{J x C}{S_{x} h_{o}}+6,76\left(\frac{0,7 f_{y}}{E}\right)}}=30923,114 \mathrm{~mm}$

- $\quad$ Finding the nominal moment value

1. Melting Condition

$$
\mathrm{Mn}=\mathrm{Mp}=\mathrm{Zx} . \text { fy }=4861,296 \mathrm{kNm}
$$

2. Nominal strength of members against bending moment

Because the structural components meet the requirements $\mathrm{Lp} \leq \mathrm{L} \leq \mathrm{L}_{\mathrm{r}}$ then the nominal strength of the structural member against the bending moment is as follows:

$\mathrm{C}_{\mathrm{b}}=\frac{12.5 M_{\max }}{2.5 M_{\max }+3 M_{A}+4 M_{B}+3 M_{C}}=0,865595$

$\left.M_{n}=C_{b} \cdot\left[M_{p}-0,7 \cdot f y \cdot S x\right) \cdot\left(\frac{L b-L p}{L r-L p}\right)\right]=1511,292 \mathrm{kNm}$

With the provision of : $M_{n} \leq M_{p}$

3. Local bending of the wing plate

Due to the flange plate is a Non-compact section then:

$\left.M_{n}=C_{b} \cdot\left[M_{p}-0,7 \cdot f y \cdot S x\right) \cdot\left(\frac{\lambda \mathrm{b}-\lambda \mathrm{p}}{\lambda \mathrm{r}-\lambda \mathrm{p}}\right)\right]=1645,847 \mathrm{kNm}$

4. Local bending of the body plate

Since the web plate is of compact cross-section, local buckling in the flange plate does not $\operatorname{occur}[19][20]$. 
- Control of bending

$$
\begin{array}{lcc}
\varnothing \mathrm{Mn} & > & \mathrm{Mu} \\
1360,163 \mathrm{kNm}> & & 1239,6045 \mathrm{kNm}
\end{array}
$$

The value of $\varnothing \mathrm{Mn}$ is greater than $\mathrm{Mu}$ then it fulfills the condition

- Sliding control

$\mathrm{V}_{\mathrm{n}}=0,6 \cdot \mathrm{f}_{\mathrm{y}} \cdot \mathrm{A}_{\mathrm{w}} \cdot \mathrm{C}_{\mathrm{v}}=285120 \mathrm{~kg}$

\begin{tabular}{|c|c|c|c|}
\hline$\Delta$ permission & $>$ & $\Delta$ happe & \\
\hline$L$ & & 5.q. $L^{4}$ & $P . L^{3}$ \\
\hline$\overline{500}$ & $>$ & $\overline{384 . E . I_{X}}+$ & $\overline{\text { 48.E. } I_{X}}$ \\
\hline
\end{tabular}

$\begin{array}{lll}\varnothing \mathrm{Vn} & > & \mathrm{Vu} \\ 256608 \mathrm{~kg} & > & 23056,12 \mathrm{~kg}\end{array}$

The value of $\varnothing \mathrm{Vn}$ is greater than $\mathrm{Vu}$ then it fulfills the condition

\section{- Control against deflection}

The value of $\Delta$ permission is greater than occurs then it fulfills the conditions

The weight of the bridge beam in this design is $21760.2 \mathrm{~kg}$

\section{Base Plate Planning}

\section{Technical Data}

Case of column with vertical load and moment (large eccentricity)

$\mathrm{Pu} \quad=6643,01 \mathrm{~kg} \quad=66430,1 \mathrm{~N}$

$\mathrm{Mu} \quad=14805,09 \mathrm{kgm}$

Tried using profile WF 350x350x19x19 :

Tried $\mathrm{B}=500 \mathrm{~mm}$, dan $\mathrm{N}=500 \mathrm{~mm}$

$$
\begin{array}{ll}
\mathrm{A}_{1} & =250000 \mathrm{~mm}^{2} \\
\mathrm{e} & =\frac{M u}{P u}=\frac{14805,09}{6643,01}=2,228672 \mathrm{~m}=2228,67 \mathrm{~mm}>\frac{N}{2}=\frac{500}{2}=250 \mathrm{~mm} \\
\mathrm{f}_{1.2} & =\frac{P}{B x N} \pm \frac{M x c}{I}==\frac{6643,01}{500 \times 500} \pm \frac{14805090 \times 250}{5208333333}=-0,2657 \pm 0,71064 \\
\mathrm{f}_{1} & =-0,4449 \\
\mathrm{f}_{2} & =0,9764
\end{array}
$$

Try column with size $600 \times 600 \mathrm{~mm} \rightarrow \mathrm{A}_{2}=360000 \mathrm{~mm}^{2}$

$$
\begin{aligned}
\frac{A 2}{A 1} & =\frac{360000}{250000}=1,44 \mathrm{~mm} \\
\mathrm{f}_{\mathrm{p}} & =0,85 \times \varphi_{\mathrm{c}} \times \mathrm{f}^{\prime} \mathrm{c} \sqrt{\frac{A 2}{A 1}} \\
& =0,85 \times 0,6 \times 20 \times 1,2 \\
& =12,24 \mathrm{MPa}
\end{aligned}
$$



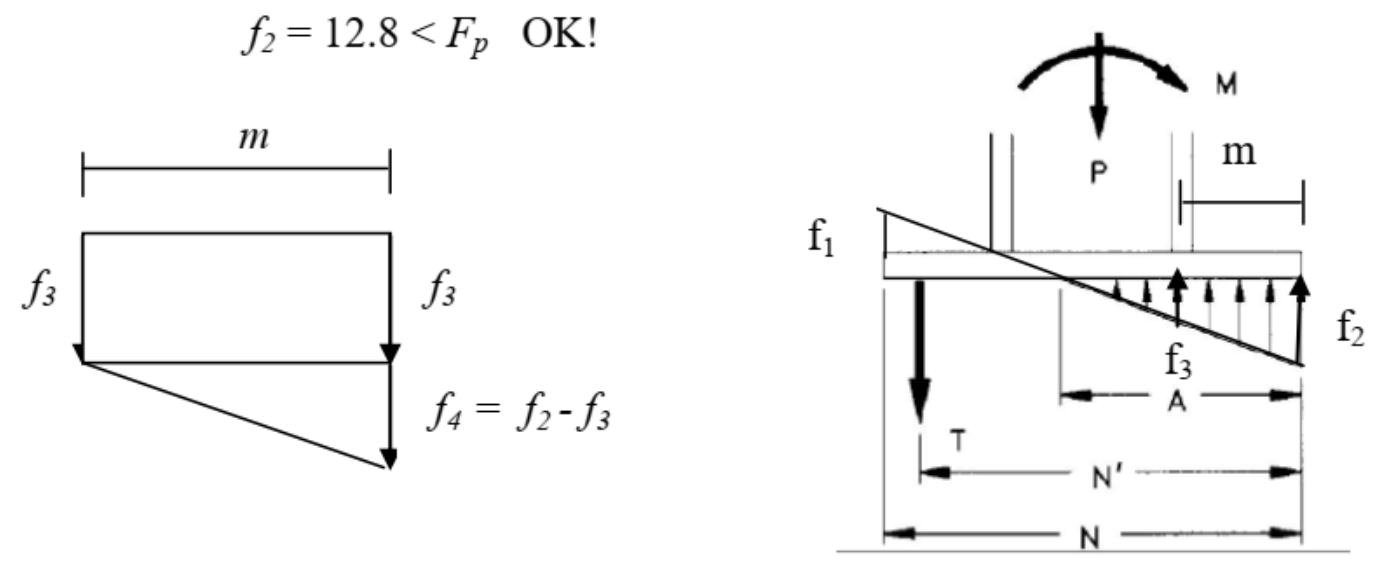

Figure 4. Column With Load

\section{Determining the Plate Thickness}

$$
\begin{array}{ll}
\mathrm{m} & =\frac{N-0,95 d}{2}=\frac{350-(0,95 \times 350)}{2}=83,75 \mathrm{~mm} \\
\mathrm{n} & =\frac{B-0,8 b f}{2}=\frac{350-(0,8 \times 357)}{2}=107,2 \mathrm{~mm}
\end{array}
$$

\section{Neutral Line}

$$
\begin{aligned}
\mathrm{A} & =\frac{f 2}{f 2+f 1} \times \mathrm{N} \quad=\frac{0,97636472}{0,97636472+0,44} \times 500=343,70 \\
\mathrm{f}_{3} \quad & =\frac{A-m}{A} \times \mathrm{f}_{2} \quad=\frac{343,70-83,75}{343,70} \times 0,97636472 \quad=0,738454 \\
\mathrm{f}_{4} \quad & =\mathrm{f}_{2}-\mathrm{f}_{3} \\
& =0,97636472-0,738454 \\
& =0,237911 \\
\mathrm{M}_{\mathrm{plu}} \quad & =\left(\frac{1}{2} \times \mathrm{f}_{3}+\frac{1}{3} \mathrm{Xf}_{4}\right) \mathrm{m}^{2} \times \mathrm{B} \\
& =\left(\frac{1}{2} \times 0,738454+\frac{1}{3} \times 0,237911\right) 83,75^{2} \times 500 \\
& =1503480 \mathrm{~mm}
\end{aligned}
$$

Plate moment capacity

$\mathrm{Mn} \quad=\varphi_{\mathrm{y}} \mathrm{Zf_{ \textrm {y } }}=\varphi_{\mathrm{y}}\left(\frac{1}{4} \mathrm{~B} \mathrm{t}_{\mathrm{p}}^{2}\right) \times \mathrm{f}_{\mathrm{y}}=0,9\left(\frac{1}{4} \mathrm{~B} \mathrm{t}_{\mathrm{p}}^{2}\right) \times \mathrm{f}_{\mathrm{y}}=\mathrm{M}_{\text {plu }}$

$t_{p}=\sqrt{\frac{4 M_{p l u}}{0,9 B f y}}=\sqrt{\frac{4 \times 1503480}{0,9 \times 500 \times 240}}=7,46 \rightarrow 8 \mathrm{~mm}$

Conclusion

Size Plate 500 x 500 × 8 mm

Column Size 600 x $600 \mathrm{~mm}$

\section{Conclusion and Suggestion}

\subsection{Conclusion}


The overall results of the calculations that have been carried out can be drawn as follows:

From the results of the planning of the WF steel roof structure on the factory warehouse construction project in Pamekasan, it was obtained planning data: Gording using Profile C 125x50x40x4,5. Trekstang uses $8 \mathrm{~mm}$ diameter, Wind ties use 10mm diameter steel, Rafter uses WF 350x350x19x19 profile, column uses WF 350x350x19x19 profile, 8 pieces A325 bolts with $22 \mathrm{~mm}$ diameter, Hoist Crane Beam uses IWF Bulit-Up beam with 600x1144x18x22 profile, Base Plate uses a size of 500x500x8mm with a column of $600 \times 600$.

\section{References}

[1] M. Z. Rozikin, Warsito, dan B. Suprapto, "Studi Perencanaan Struktur Baja Pada Bangunan Gedung Kampus Stkip Al Hikmah Surabaya,” J. Rekayasa Sipil, vol. 8, no. 1, hal. 23-33, 2020.

[2] I. L. Belakang, “Asesmen Struktur Gedung Kantor Camat Nongsa Batam,” vol. II, no. $2,2014$.

[3] F. Phiegiarto, J. E. Tjanniadi, H. Santoso, dan I. Muljati, "Perencanaan Elemen Struktur Baja Berdasarkan Sni 1729 : 2015,” Univ. Kristen Petra, hal. 1-8, 2015.

[4] W. G. Edi, P. M. D. Septyani, T. Sri, dan W. Hardi, "Redesain Struktur Gedung Kuliah Umum Fakultas Teknik Universitas Diponegoro Menggunakan Konstruksi Baja Berdasarkan Sni 1729-2015 Dan Sni 7972-2013,” J. Karya Tek. Sipil, vol. 6, no. Volume 6, Nomor 3, Tahun 2017, hal. 182-196, 2017.

[5] C. A. Sarasti, "Perencanaan Gedung Perkuliahan Fakultas Perikanan Dan Ilmu Kelautan Universitas Brawijaya Mengunakan Struktur Baja Komposit Dengan Etode Lrfd Berdasarkan SNI-03-1729-2002,”Ekp, vol. 13, no. 3, hal. 1576-1580, 2015.

[6] M. Simamora, D. Trisnoyuwono, dan A. H. Muda, "Dampak Kerusakan Dini Perkerasan Jalan terhadap Kerugian Aspek Finansial," Media Komun. Tek. Sipil, vol. 24, no. 2, hal. 184, 2019.

[7] R. A. Puspitadewi, “Analisis Dan Desain Struktur Baja Pada Gedung Bertingkat 4 Berdasarkan Sni 03-1729-2002 Analysis And Design Steel Structure At Story Building 4 Of Sni 03-1729-2002,” 2011.

[8] S. Baja, U. Bangunan, dan G. Sni, "Perencanaan sambungan kaku balok - kolom tipe end plate menurut tata cara perencanaan struktur baja untuk bangunan gedung (sni 03 - 
1729 - 2002) menggunakan microsoft excel 2013,” 2014.

[9] Badan Standarisasi Nasional, "Spesifikasi untuk bangunan gedung baja struktural Badan Standardisasi Nasional (SNI 1729:2015),” Bandung, hal. 1-289, 2015.

[10] J. T. Sipil, F. Teknik, dan U. N. Semarang, "Perencanaan ulang pembangunan gedung universitas widya dharma klaten jawa tengah," 2015.

[11] M. Khafis, "Perencanaan Struktur Baja Pada Bangunan Tujuh Lantai Sebagai Hotel," Univ. Sebel. Maret. Surakarta, 2016.

[12] Maradhika Fauzy, "Perencanaan Struktur Baja Gedung Hotel NEO," Sipil, Jur. Tek. Tek. Fak. Semarang, Univ. Negeri, 2016.

[13] T. C. Morphology, “Analisis Konfigurasi Bentuk Pengaku Pada Perencanaan Struktur Gedung Rangka Baja Dengan Pengaku Konsentrik,” hal. 1-13.

[14] SNI 03-1729-2002, "Tata Cara Perencanaan Struktur Baja untuk Bangunan Gedung," Standar Nas. Indones., 2002.

[15] B. Spesification, A. Lrfd, Dan O. A. Hamid, "Perencanaan Gedung Perkantoran / Perkuliahanuniversitas Muhammadiyah Malang Denganbalok-Lantai Komposit Dan Kolom Baja,” No. 99520162, 2014.

[16] BSN, “Standar Nasional Indonesia,” Badan Standar Nas., 2017.

[17] S. Woo, Reliability Design of Mechanical Systems, no. January. 2017.

[18] S. Wunda, A. Z. Johannes, R. K. Pingak, dan A. S. Ahab, “Analisis Tegangan, Regangan Dan Deformasi Crane Hook Dari Material Baja Aisi 1045 Dan Baja St 37 Menggunakan Software Elmer,” J. Fis. Fis. Sains dan Apl., vol. 4, no. 2, hal. 131-137, 2019.

[19] J. Triyanto, Z. Djauhari, dan M. Olivia, “Tinjauan Gaya Momen Pada Tiap Batang,” vol. 01, no. 01, hal. 33-42, 2018.

[20] J. T. Sipil, F. Teknik, I. Teknologi, dan S. Nopember, “Analisa Perbandingan Model Keruntuhan Profil Hexagonal,” 2018. 